\title{
APPLICATION OF A GREEN'S FUNCTION METHOD TO HEAT CONDUCTION PROBLEMS IN MULTI-LAYERED CYLINDERS
}

\author{
Urszula Siedlecka, Stanistaw Kukla \\ Institute of Mathematics, Czestochowa University of Technology \\ Częstochowa, Poland \\ urszula.siedlecka@im.pcz.pl,stanislaw.kukla@im.pcz.pl
}

\begin{abstract}
In this paper, the Green's functions which are used in solving the heat conduction problem in a composite cylinder are derived. The functions are obtained by the solution of one-dimensional eigenproblems and are presented in the form of eigenfunction series. The temperature in the cylinder as a function of time and space coordinates are expressed by the Green's functions. A numerical example is presented.
\end{abstract}

Keywords: heat conduction, Green's function, composite cylinder

\section{Introduction}

The heat conduction problem in a composite circular cylinder has been considered by Lu et al. in paper [1]. The problem has been solved by applying the Laplace transform and the closed form solution as the real part of a function is given. Likewise, in papers $[2,3]$, the solution of the heat conduction problem in a cylinder was obtained by using the Laplace transform. The inverse transform has been numerically determined. However, the numerical computations of the inverse transform often leads to numerical instabilities and for this reason the seeking of new methods is purposeful. The other approach to solving the problem is the use of the properties of Green's functions which correspond to the initial boundary problem of heat conduction in the layers of the considered composite cylinder. Applications of the Green's functions to various problems of heat conduction are presented in the book by Beck et al. [4], in the book by Duffy [5] and in the book by Özişik [6]. The Green's function method to the heat conduction in a composite two-layered cylinder has been applied in reference [7].

In this paper the Green's functions applying in the problems of the heat conduction in finite, composite multi-layered cylinders are derived. The functions are obtained by solving the heat conduction differential equation with homogenous boundary conditions of the second and third kind. An example of application of the Green's functions to the problem of heat conduction in radial direction of a two-layered cylinder is given. The temperature in the cylinder as a time function is numerically computed. 


\section{Derivation of Green's functions}

The Green's functions for the problems of heat conduction satisfy the following differential equation

$$
\nabla^{2} G-\frac{1}{\alpha} \frac{\partial G}{\partial t}=\frac{1}{r} \delta(r-\rho) \delta(z-\zeta) \delta(t-\tau) \delta(\theta-\varphi)
$$

where $\nabla^{2}=\frac{\partial^{2}}{\partial r^{2}}+\frac{1}{r} \frac{\partial}{\partial r}+\frac{1}{r^{2}} \frac{\partial^{2}}{\partial \theta^{2}}+\frac{\partial^{2}}{\partial z^{2}}$ is the laplacian in cylindrical coordinates, $\alpha$ is the diffusion coefficient, $r, \theta, z$ are the space coordinates in cylindrical coordinate system and $t$ is time (Fig. 1).

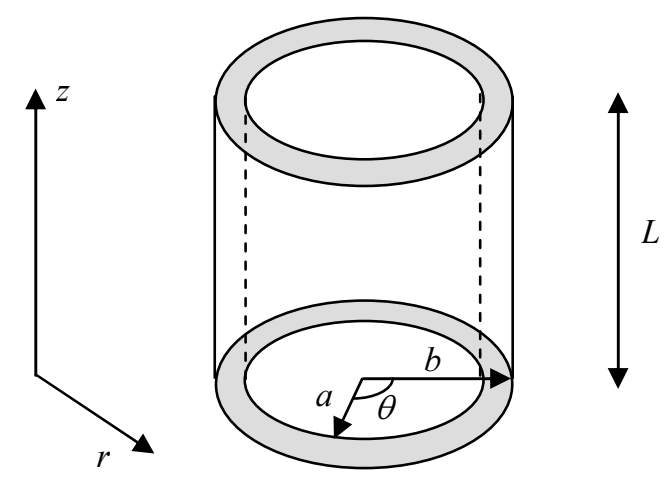

Fig. 1. The sketch of the considered cylindrical region

We assume the homogeneous boundary conditions in the form:

$$
\begin{aligned}
& a_{1} \frac{\partial G}{\partial r}-a_{2} G=0 \text { for } r=a \\
& b_{1} \frac{\partial G}{\partial r}-b_{2} G=0 \text { for } r=b \\
& \gamma_{1} \frac{\partial G}{\partial z}-\gamma_{2} G=0 \text { for } z=0 \\
& v_{1} \frac{\partial G}{\partial z}-v_{2} G=0 \text { for } z=L
\end{aligned}
$$

Moreover, the function zero initial condition held

$$
G(0, r, \theta, z ; \tau, \rho, \varphi, \zeta)=0
$$


We search the solution of the initial - boundary problem (1)-(6) in the form

$$
G(t, r, \theta, z ; \tau, \rho, \varphi, \zeta)=\sum_{n=0}^{\infty} \frac{1}{\kappa_{n}} g_{n}(t, r, z ; \tau, \rho, \zeta) \cos n(\theta-\varphi)
$$

where $\kappa_{0}=2 \pi$ and $\kappa_{n}=\pi$ for $n>0$. Substituting (7) into equation (1), we obtain differential equation in the form

$$
\frac{\partial^{2} g_{n}}{\partial r^{2}}+\frac{1}{r} \frac{\partial g_{n}}{\partial r}-\frac{n^{2}}{r^{2}} g_{n}+\frac{\partial^{2} g_{n}}{\partial z^{2}}-\frac{1}{\alpha} \frac{\partial g_{n}}{\partial t}=\frac{1}{r} \delta(r-\rho) \delta(z-\zeta) \delta(t-\tau)
$$

The function $g_{n}(t, r, z ; \tau, \rho, \zeta)$ can be expressed in the form of the product of two $1 D$ Green's functions [4]

$$
g_{n}(t, r, z ; \tau, \rho, \zeta)=R_{n}(t, r ; \tau, \rho) \cdot Z(t, z ; \tau, \zeta)
$$

The function $R_{n}$ is a solution of a boundary problem:

$$
\begin{gathered}
\left(\frac{\partial^{2}}{\partial r^{2}}+\frac{1}{r} \frac{\partial}{\partial r}-\frac{n^{2}}{r^{2}}\right) R_{n}+\frac{1}{r} \delta(r-\rho) \delta(t-\tau)=\frac{1}{\alpha} \frac{\partial R_{n}}{\partial t} \\
a_{1} \frac{\partial R_{n}}{\partial r}-a_{2} R_{n}=0 \text { for } r=a \\
b_{1} \frac{\partial R_{n}}{\partial r}-b_{2} R_{n}=0 \text { for } r=b
\end{gathered}
$$

while the function $Z$ is a solution of the following boundary problem:

$$
\begin{gathered}
\frac{\partial^{2} Z}{\partial z^{2}}+\delta(z-\zeta) \delta(t-\tau)=\frac{1}{\alpha} \frac{\partial Z}{\partial t} \\
\gamma_{1} \frac{\partial Z}{\partial z}-\gamma_{2} Z=0 \text { for } z=0 \\
v_{1} \frac{\partial Z}{\partial z}-v_{2} Z=0 \text { for } z=L
\end{gathered}
$$

We seek the function $R_{n}$ in the form of a series of eigenfunctions $\phi_{n k}(r)$ of the homogeneous problem corresponding to the boundary problem (10)-(12). The sought functions $\phi_{n k}(r)$ satisfy the equation 


$$
\left(\frac{\partial^{2}}{\partial r^{2}}+\frac{1}{r} \frac{\partial}{\partial r}-\frac{n^{2}}{r^{2}}\right) \phi_{n k}=\lambda^{2} \phi_{n k}
$$

where $\lambda$ is the separation constant. Moreover, the functions $\phi_{n k}(r)$ satisfy the boundary conditions analogous to the given by equations (11)-(12). We assume the functions $\phi_{n k}$ in the form

$$
\phi_{n k}(r)=A_{12} J_{n}\left(\lambda_{n k} r\right)-A_{11} Y_{n}\left(\lambda_{n k} r\right)
$$

where $\lambda_{n k}$ are roots of the eigenvalue equation

$$
A_{11} A_{22}-A_{12} A_{21}=0
$$

where

$$
\begin{aligned}
& A_{11}=a_{1} \lambda J_{n}^{\prime}(\lambda a)-a_{2} J_{n}(\lambda a), \quad A_{12}=a_{1} \lambda Y_{n}^{\prime}(\lambda a)-a_{2} Y_{n}(\lambda a) \\
& A_{21}=b_{1} \lambda J_{n}^{\prime}(\lambda b)-b_{2} J_{n}(\lambda b), \quad A_{22}=b_{1} \lambda Y_{n}^{\prime}(\lambda b)-b_{2} Y_{n}(\lambda b)
\end{aligned}
$$

The functions $\phi_{n k}$ for each $n$, create a sequence of orthogonal functions, i.e. the functions satisfy the condition

$$
\int_{a}^{b} r \phi_{n k}(r) \phi_{n k^{\prime}}(r) d r= \begin{cases}0 & \text { for } k^{\prime} \neq k \\ N_{n k}^{R} & \text { for } k^{\prime}=k\end{cases}
$$

where

$$
N_{n k}^{R}=\int_{a}^{b} r\left(\phi_{n k}(r)\right)^{2} d r
$$

Using the functions $\phi_{n k}$, the solution of the problem (10)-(12) can be written in the form

$$
R_{n}(t, r ; \tau, \rho)=\sum_{k=1}^{\infty} \frac{\phi_{n k}(r) \phi_{n k}(\rho)}{N_{n k}^{R}} e^{-\lambda_{n k}^{2} \alpha(t-\tau)}
$$

Similarly, the solution of the boundary problem (13)-(15) we find in the form

$$
Z(t, z ; \tau, \zeta)=\sum_{k=1}^{\infty} \frac{\psi_{k}(z) \psi_{k}(\zeta)}{N_{k}^{Z}} e^{-\beta_{k}^{2} \alpha(t-\tau)}
$$

The eigenvalues $\beta_{k}$ are the roots of equation

$$
\left(\gamma_{1} v_{2}-\gamma_{2} v_{1}\right) \beta \cos \beta L+\left(\gamma_{1} v_{1} \beta^{2}+\gamma_{2} v_{2}\right) \sin \beta L=0
$$


and the functions $\psi_{k}$ have the form

$$
\psi_{k}(z)=\gamma_{1} \beta_{k} \cos \beta_{k} z+\gamma_{2} \sin \beta_{k} z
$$

and

$$
N_{k}^{Z}=\int_{0}^{L}\left(\psi_{k}(z)\right)^{2} d z
$$

Finally, the Green's function $G(t, r, \theta, z ; \tau, \rho, \varphi, \zeta)$ of the problem (1)-(6) is given by equation (7) where the function $g_{n}(t, r, z ; \tau, \rho, \zeta)$ is expressed by equation (9) and the functions $R_{n}(t, r ; \tau, \rho), Z(t, z ; \tau, \zeta)$ are presented by (24) and (25), respectively.

The Green's function for axisymmetric heat conduction problem can be obtained assuming $n=0$ in an equation (8). That way the Green's function for axisymmetric heat conduction problems has the form

$$
G(t, r, z ; \tau, \rho, \zeta)=\sum_{k=1}^{\infty} \sum_{l=1}^{\infty} \frac{\phi_{0 k}(r) \phi_{0 k}(\rho)}{N_{0 k}^{R}} \frac{\psi_{l}(z) \psi_{l}(\zeta)}{N_{l}^{Z}} e^{\left(-\lambda_{0 k}^{2}-\beta_{l}^{2}\right) \alpha(t-\tau)}
$$

where $\phi_{0 k}, N_{0 k}^{R}, \psi_{k}$, and $N_{k}^{Z}$ are given by equations (18), (23), (27) and (28), respectively.

The assumption, that the heat conduction is in the radial direction only, leads to the Green's function in the form

$$
\begin{aligned}
& G(t, r ; \tau, \rho)= \\
& =\sum_{k=1}^{\infty} \frac{\left(A_{12} J_{0}\left(\lambda_{0 k} r\right)-A_{11} Y_{0}\left(\lambda_{0 k} r\right)\right)\left(A_{12} J_{0}\left(\lambda_{0 k} \rho\right)-A_{11} Y_{0}\left(\lambda_{0 k} \rho\right)\right)}{N_{0 k}^{R}} e^{-\lambda_{0 k}^{2} \alpha(t-\tau)}
\end{aligned}
$$

where $A_{12}$ and $A_{11}$ are given by equations (20) for $n=0$.

\section{The Green's functions for the problem of heat conduction in $m$-layered composite cylinder}

To solve the problem of heat conduction in the composite $m$-layered cylinder by the aid of the GF method, we use the Green's functions satisfying boundary conditions which can be obtained by suitable adoption of the coefficients, occurring in boundary conditions (2)-(5). For example, to observe the heat conduction in the radial direction in a two-layered hollow cylinder $(\mathrm{m}=2)$, we assume in boundary 
conditions (11)-(12) and in equation (30): $a_{1}=\alpha_{1}, a_{2}=\alpha_{+}, b_{1}=1, b_{2}=0, a=r_{0}$, $b=r_{1}, \alpha=k_{1}$, to obtain the function $G_{1}$ and $a_{1}=1, a_{2}=0, b_{1}=\alpha_{2}, b_{2}=\alpha_{\infty}$, $a=r_{1}, b=r_{2}, \alpha=k_{2}$, to obtain the function $G_{2}$. The boundary conditions, eigenfunctions $\phi_{0 k}$, eigenvalue equations and norms of eigenfunctions $N_{0 k}^{R}$ for that problem are presented below:

A. Boundary conditions: $\frac{\partial G_{1}}{\partial r}-\gamma_{0} G_{1}=0$ for $r=r_{0}, \frac{\partial G_{1}}{\partial r}=0$ for $r=r_{1} \quad\left(\gamma_{1}=\frac{\alpha_{+}}{\alpha_{1}}\right)$

(a) eigenfunctions:

$$
\phi_{0 k}^{[1]}=J_{0}\left(r \lambda_{0 k}^{[1]}\right) Y_{1}\left(r_{1} \lambda_{0 k}^{[1]}\right)-Y_{0}\left(r \lambda_{0 k}^{[1]}\right) J_{1}\left(r_{1} \lambda_{0 k}^{[1]}\right)
$$

(b) eigenequation:

$$
\begin{aligned}
\left(\lambda_{0 k}^{[1]} J_{1}\left(r_{0} \lambda_{0 k}^{[1]}\right)\right. & \left.+\gamma_{1} J_{0}\left(r_{0} \lambda_{0 k}^{[1]}\right)\right) Y_{1}\left(r_{1} \lambda_{0 k}^{[1]}\right)- \\
& -\left(\lambda_{0 k}^{[1]} Y_{1}\left(r_{0} \lambda_{0 k}^{[1]}\right)+\gamma_{1} Y_{0}\left(r_{0} \lambda_{0 k}^{[1]}\right)\right) J_{1}\left(r_{1} \lambda_{0 k}^{[1]}\right)=0
\end{aligned}
$$

(c) norms of eigenfunctions:

$$
\begin{aligned}
& N_{0 k}^{R}=\frac{2}{\pi^{2}\left(\lambda_{0 k}^{[1]}\right)^{2}}- \frac{1}{2} r_{0}^{2} J_{1}^{2}\left(r_{1} \lambda_{0 k}^{[1]}\right)\left(Y_{0}^{2}\left(r_{0} \lambda_{0 k}^{[1]}\right)+Y_{1}^{2}\left(r_{0} \lambda_{0 k}^{[1]}\right)\right)+ \\
&+r_{0}^{2} J_{1}\left(r_{1} \lambda_{0 k}^{[1]}\right) Y_{1}\left(r_{1} \lambda_{0 k}^{[1]}\right)\left[J_{0}\left(r_{0} \lambda_{0 k}^{[1]}\right) Y_{0}\left(r_{0} \lambda_{0 k}^{[1]}\right)+J_{1}\left(r_{0} \lambda_{0 k}^{[1]}\right) Y_{1}\left(r_{0} \lambda_{0 k}^{[1]}\right)\right]- \\
&-\frac{1}{2} r_{0}^{2}\left(J_{0}^{2}\left(r_{0} \lambda_{0 k}^{[1]}\right)+J_{1}^{2}\left(r_{0} \lambda_{0 k}^{[1]}\right)\right) Y_{1}^{2}\left(r_{1} \lambda_{0 k}^{[1]}\right)
\end{aligned}
$$

B. Boundary conditions: $\frac{\partial G_{i}}{\partial r}=0$ for $r=r_{i-1}, \frac{\partial G_{i}}{\partial r}=0$ for $r=r_{i}, i=2,3, \ldots, m-1$

(a) eigenfunctions:

$$
\phi_{0 k}^{[i]}=J_{0}\left(r \lambda_{0 k}^{[i]}\right) Y_{1}\left(r_{i} \lambda_{0 k}^{[i]}\right)-Y_{0}\left(r \lambda_{0 k}^{[i]}\right) J_{1}\left(r_{i} \lambda_{0 k}^{[i]}\right)
$$

(b) eigenequation:

$$
J_{1}\left(r_{i-1} \lambda_{0 k}^{[i]}\right) Y_{1}\left(r_{i} \lambda_{0 k}^{[i]}\right)-Y_{1}\left(r_{i-1} \lambda_{0 k}^{[i]}\right) J_{1}\left(r_{i} \lambda_{0 k}^{[i]}\right)=0
$$

(c) norms of eigenfunctions: 


$$
\begin{aligned}
& N_{0 k}^{R}=\frac{2}{\pi^{2}\left(\lambda_{0 k}^{[i]}\right)^{2}}-\frac{1}{2} r_{i-1}^{2} J_{1}^{2}\left(r_{i} \lambda_{0 k}^{[i]}\right)\left(Y_{0}^{2}\left(r_{i-1} \lambda_{0 k}^{[i]}\right)+Y_{1}^{2}\left(r_{i-1} \lambda_{0 k}^{[i]}\right)\right)+ \\
& +r_{i-1}{ }^{2} J_{1}\left(r_{i} \lambda_{0 k}^{[i]}\right) Y_{1}\left(r_{i} \lambda_{0 k}^{[i]}\right)\left[J_{0}\left(r_{i-1} \lambda_{0 k}^{[i]}\right) Y_{0}\left(r_{i-1} \lambda_{0 k}^{[i]}\right)+J_{1}\left(r_{i-1} \lambda_{0 k}^{[i]}\right) Y_{1}\left(r_{i-1} \lambda_{0 k}^{[i]}\right)\right]- \\
& \quad-\frac{1}{2} r_{i-1}{ }^{2}\left(J_{0}^{2}\left(r_{i-1} \lambda_{0 k}^{[i]}\right)+J_{1}^{2}\left(r_{i-1} \lambda_{0 k}^{[i]}\right)\right) Y_{1}^{2}\left(r_{i} \lambda_{0 k}^{[i]}\right)
\end{aligned}
$$

C. Boundary conditions: $\frac{\partial G_{m}}{\partial r}=0$ for $r=r_{m-1}, \frac{\partial G_{m}}{\partial r}-\gamma_{m} G_{m}=0$ for $r=r_{m}\left(\gamma_{1}=\frac{\alpha_{\infty}}{\alpha_{m}}\right)$

(a) eigenfunctions:

$$
\phi_{0 k}^{[m]}=J_{0}\left(r \lambda_{0 k}^{[m]}\right) Y_{1}\left(r_{m-1} \lambda_{0 k}^{[m]}\right)-Y_{0}\left(r \lambda_{0 k}^{[m]}\right) J_{1}\left(r_{m-1} \lambda_{0 k}^{[m]}\right)
$$

(b) eigenequation:

$$
\begin{aligned}
\left(\lambda_{0 k}^{[m]} Y_{1}\left(r_{m} \lambda_{0 k}^{[m]}\right)-\gamma_{m} Y_{0}\left(r_{m} \lambda_{0 k}^{[m]}\right)\right) J_{1}\left(r_{m-1} \lambda_{0 k}^{[m]}\right)- & \\
& -\left(\lambda_{0 k}^{[m]} J_{1}\left(r_{m} \lambda_{0 k}^{[m]}\right)-\gamma_{m} J_{0}\left(r_{m} \lambda_{0 k}^{[m]}\right)\right) Y_{1}\left(r_{m-1} \lambda_{0 k}^{[m]}\right)=0
\end{aligned}
$$

(c) norms of eigenfunctions:

$$
\begin{aligned}
& N_{0 k}^{R}=\frac{2}{\pi^{2}\left(\lambda_{0 k}^{[m]}\right)^{2}}-\frac{1}{2} r_{m-1}^{2} J_{1}^{2}\left(r_{m} \lambda_{0 k}^{[m]}\right)\left(Y_{0}^{2}\left(r_{m-1} \lambda_{0 k}^{[m]}\right)+Y_{1}^{2}\left(r_{m-1} \lambda_{0 k}^{[m]}\right)\right)+ \\
& +r_{m-1}^{2} J_{1}\left(r_{m} \lambda_{0 k}^{[m]}\right) Y_{1}\left(r_{m} \lambda_{0 k}^{[m]}\right)\left[J_{0}\left(r_{m-1} \lambda_{0 k}^{[m]}\right) Y_{0}\left(r_{m-1} \lambda_{0 k}^{[m]}\right)+J_{1}\left(r_{m-1} \lambda_{0 k}^{[m]}\right) Y_{1}\left(r_{m-1} \lambda_{0 k}^{[m]}\right)\right] \\
& -\frac{1}{2} r_{m-1}^{2}\left(J_{0}^{2}\left(r_{m-1} \lambda_{0 k}^{[m]}\right)+J_{1}^{2}\left(r_{m-1} \lambda_{0 k}^{[m]}\right)\right) Y_{1}^{2}\left(r_{m} \lambda_{0 k}^{[m]}\right)
\end{aligned}
$$

\section{Numerical example}

We determine the temperature in the two-layered cylinder by using properties of the Green's functions which are derived in the previous section. The temperatures $T_{1}$ and $T_{2}$ in the cylinder layers, submitted in the paper [7], are given in the form

$$
\begin{gathered}
T_{1}(t, r)=k_{1} \int_{0}^{t}\left[r_{1} y(\tau) G_{1}\left(t, r ; \tau, r_{1}\right)+r_{0} \gamma_{1} T_{+}(\tau) G_{1}\left(t, r ; \tau, r_{0}\right)\right] d \tau \text { for } r \in\left[r_{0}, r_{1}\right] \text { (39) } \\
T_{2}(t, r)=k_{2} \int_{0}^{t}\left[r_{2} \gamma_{2} T_{\infty}(\tau) G_{2}\left(t, r ; \tau, r_{2}\right)-r_{1} \frac{\alpha_{1}}{\alpha_{2}} y(\tau) G_{2}\left(t, r ; \tau, r_{1}\right)\right] d \tau \text { for } r \in\left[r_{1}, r_{2}\right]
\end{gathered}
$$


The function $y(\tau)$ is a solution of the Volterra integral equation of the first kind [7]:

$$
\int_{0}^{t} K(t, \tau) y(\tau) d \tau=F(t)
$$

where

$$
K(t, \tau)=k_{1} r_{1} G_{1}\left(t, r_{1} ; \tau, r_{1}\right)+k_{2} r_{1} \frac{\alpha_{1}}{\alpha_{2}} G_{2}\left(t, r_{1} ; \tau, r_{1}\right)
$$

and

$$
F(t)=\int_{0}^{t}\left[k_{2} r_{2} \gamma_{2} T_{\infty}(\tau) G_{2}\left(t, r_{1} ; \tau, r_{2}\right)-k_{1} r_{0} \gamma_{1} T_{+}(\tau) G_{1}\left(t, r_{1} ; \tau, r_{0}\right)\right] d \tau
$$

A numerical procedure to solution of the equation (42) is presented in paper [7].

The numerical calculations of the temperatures $T_{1}$ and $T_{2}$ in the cylinder are performed for the following geometrical and physical data: $r_{0}=0,04 \mathrm{~m}, r_{1}=0,045 \mathrm{~m}$, $r_{2}=0,06 \mathrm{~m}, \quad k_{1}=7,5 \cdot 10^{-7} \mathrm{~m}^{2} / \mathrm{s}, \quad k_{2}=1,4 \cdot 10^{-6} \mathrm{~m}^{2} / \mathrm{s}, \quad \alpha_{1}=0,08 \mathrm{~W} /(\mathrm{m} \mathrm{K})$, $\alpha_{2}=0,04 \mathrm{~W} /(\mathrm{m} \mathrm{K}), \alpha_{+}=9,0 \mathrm{~W} /\left(\mathrm{m}^{2} \mathrm{~K}\right), \alpha_{\infty}=20,0 \mathrm{~W} /\left(\mathrm{m}^{2} \mathrm{~K}\right)$. Boundary temperatures are assumed as: $T_{\infty}(t)=25^{\circ} \mathrm{C}, T_{+}(t)=60+30 \cos (0,05 t)$.

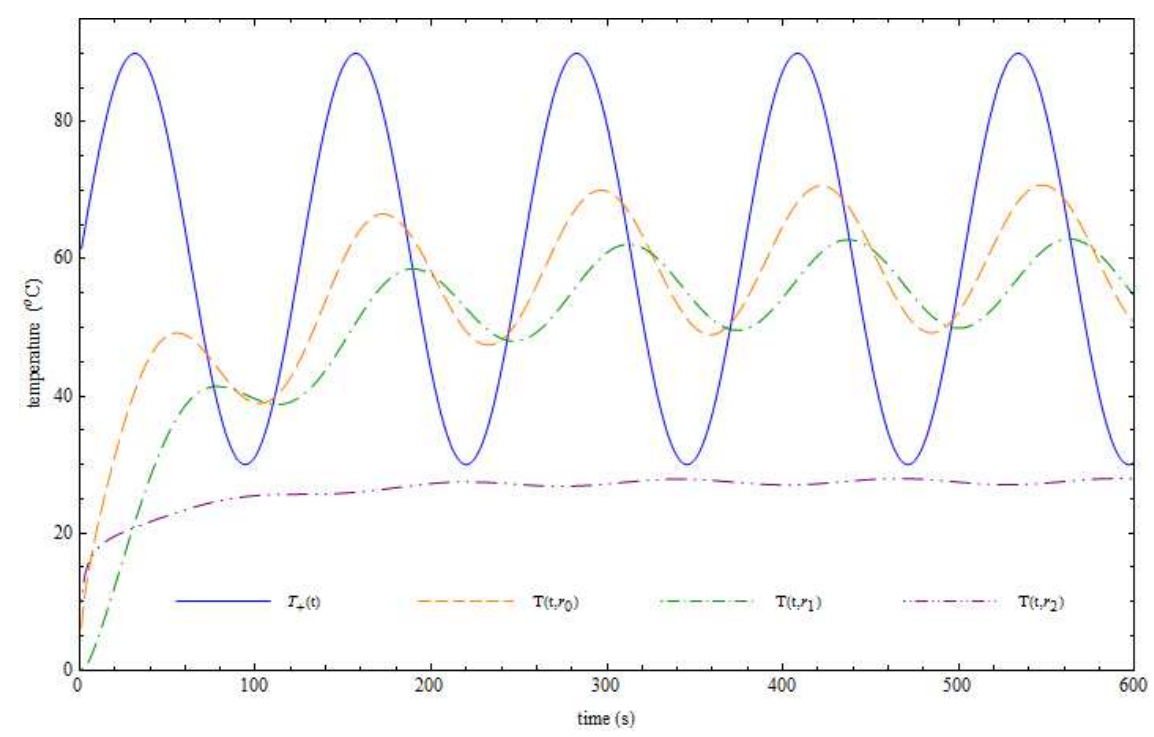

Fig. 2. Variations of the temperature versus time in the inner surroundings of the cylinder and at the points: $r=r_{0}, r=r_{1}, r=r_{2}$

The time history of temperatures $T(t, r)$ at $r=r_{0}, r=r_{1}, r=r_{2}$ and temperature $T_{+}(t)$ for $0 \leq t \leq 600$, is presented in Figure 2. The periodical changes of the 
temperature in the cylinder layers are caused by the change of the temperature in the inner surroundings of the cylinder. It can be observed, that temperature at the outer boundary of the cylinder for the used diffusion coefficient, changes slightly over time.

\section{Conclusions}

The Green's functions for problems of the heat conduction in a composite hollow cylinder are determined. The functions are presented in the form of eigenfunctions series. There are three cases of boundary conditions considered in the radial direction at the surfaces of the layers and the inner and outer surfaces of the cylinder: Robin-Neumann, Neumann-Neumann and Neumann-Robin conditions. The temperature in the layers of the cylinder with aid of the Green's functions in an analytical form is expressed. Assuming a periodic change of the temperature in the inner surroundings of the cylinder and a constant temperature in the outer surroundings, variations of the temperature versus time in the two-layered cylinder is numerically determined. Although the numerical computation deals with the two-layered cylinder, the presented solution can be used in numerical simulation of the temperature in the multi-layered cylinder.

\section{References}

[1] Lu X., Tervola P., Viljanen M., Transient analytical solution to heat conduction in composite circular cylinder, International Journal of Heat and Mass Transfer 2006, 49, 341-348.

[2] Nezhad Y.R., Asemi K., Akhlaghi M., Transient solution of temperature field in functionally graded hollow cylinder with finite length using multi-layered approach, International Journal of Mechanics and Materials in Design 2011, 7, 71-82.

[3] Garbai L., Krope J., Mehes S., Bartal I., Transient heat conduction in composite systems, Proceedings of the 4th WSEAS International Conference on Heat Transfer, Thermal Engineering and Environment, Elounda, Greece, August 21-23, 2006, 372-379.

[4] Beck J.V., Cole K.D., Haji-Sheikh A., Litkouhi B., Heat Conduction Using Green's Functions, Hemisphere, Washington, DC 1992.

[5] Duffy D.G., Green's functions with applications, Chapman\&Hall/CRC, Washington, DC 2001.

[6] Özişik M.N., Heat condition, second edition, John Wiley \& Sons, New York 1993.

[7] Kukla S., Siedlecka U., Heat conduction in a two-layered hollow cylinder by Green's function method, Journal of Applied Mathematics and Computational Mechanics 2013, 12(2), 45-50.

[8] Grzymkowski R., Hetmaniok E., Słota D., Wykłady z modelowania matematycznego, Wydawnictwo Pracowni Komputerowej Jacka Skalmierskiego, Gliwice 2002.

[9] Wolfram S., The Mathematica Book, Wolfram Media, 5th ed., 2003. 